\title{
Pinhole Closure Measurements
}

\author{
D. Milam, J. E. Murray, K. G. Estabrook, \\ C. D. Boley, W. D. Sell, N. D. Nielsen, \\ R. K. Kirkwood, Y. A. Zakharenkov, \\ and B. B. Afeyan
}

This paper was prepared for submittal to the

Third Annual International Conference on

Solid State Lasers for Application (SSLA)

to Inertial Confinement Fusion (ICF)

Monterey, California

June 7-12, 1998

July 20, 1998

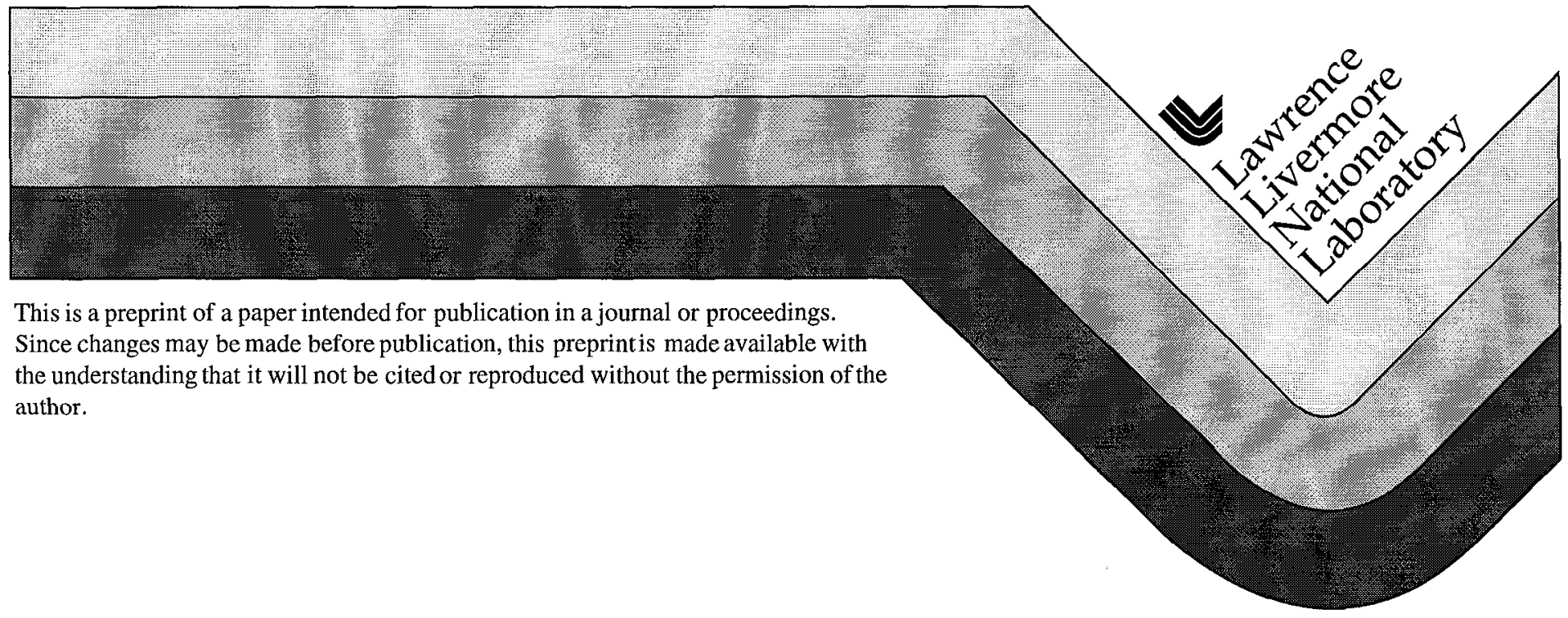


DISCLAIMER

This document was prepared as an account of work sponsored by an agency of the United States Government. Neither the United States Government nor the University of California nor any of their employees, makes any warranty, express or implied, or assumes any legal liability or responsibility for the accuracy, completeness, or usefulness of any information, apparatus, product, or process disclosed, or represents that its use would not infringe privately owned rights. Reference herein to any specific commercial product, process, or service by trade name, trademark, manufacturer, or otherwise, does not necessarily constitute or imply its endorsement, recommendation, or favoring by the United States Government or the University of California. The views and opinions of authors expressed herein do not necessarily state or reflect those of the United States Government or the University of California, and shall not be used for advertising or product endorsement purposes. 


\author{
University of California \\ Lawrence Livermore National Laboratory \\ Livermore, CA 94550 USA \\ B. B. Afeyan
}

Polymath Associates

Livermore, CA, 94550 USA

\begin{abstract}
Spatial-filter pinholes and knife-edge samples were irradiated in vacuum by $1053-\mathrm{nm}, 5-20 \mathrm{~ns}$ pulses at intensities to $500 \mathrm{GW} / \mathrm{cm}^{2}$. The knife-edge samples were fabricated of plastic, carbon, aluminum, stainless steel, molybdenum, tantalum, gold and an absorbing glass. Time-resolved two-beam interferometry with a 40-ns probe pulse was used to observe phase shifts in the expanding laser-induced plasma. For all of these materials, at any time during square-pulse irradiation, the phase shift fell exponentially with distance from the edge of the sample. The expansion was characterized by the propagation velocity $V_{2 \pi}$ of the contour for a $2 \pi$ phase shift. To within experimental error, $V_{2 \pi}$ was constant during irradiation at a particular intensity, and it increased linearly with intensity for intensities $<300 \mathrm{GW} / \mathrm{cm}^{2}$. For metal samples, $V_{2 \pi}$ exhibited an approximate $\mathrm{M}^{-0.5}$ dependence where $\mathrm{M}$ is the atomic mass. Plasmas of plastic, carbon and absorbing glass produced larger phase shifts, and expanded more rapidly, than plasmas of the heavy metals. The probe beam and interferometer were also used to observe the closing of pinholes. With planar pinholes, accumulation of on-axis plasma was observed along with the advance of plasma away from the edge of the hole. On-axis closure was not observed in square, 4-leaf pinholes.

Kcywords: lascr-induced plasma, carbon, aluminum, iron, molybdenum, tantalum, absorbing glass, pinhole closure
\end{abstract}

\title{
1. EXPERIMENTAL PROCEDURE
}

Laser pulses for sample irradiation were provided by the Optical Science Laser (OSL) at Livermore. The laser provides output of about $50 \mathrm{~J}$, in pulses with duration adjustable from 1-100 ns, in an 80-mm diameter bcam that is formed by four stages of image relaying. During these experiments, the diameter of the beam was first reduced to $30 \mathrm{~mm}$ by an inverted telescope which is part of the image relaying. Intensity in the $30-\mathrm{mm}$ beam was uniform to within about $15 \%$ over the central 20-mm region. To produce the relatively large intensities that were required for generation of plasmas, the beam diameter was reduced to either 1.4 or $2 \mathrm{~mm}$ by single-lens down magnification. Figure 1 shows the fluence distribution in the 2-mm beam. It is a slightly distorted replica of the output relay plane of the laser.

During all of the knife-edge tests that are described here, one-half of the beam was intercepted by a vertically oriented edge. The beam intensity was selected to be similar to the expected loading of the fourth-pass pinhole in the F\#31 NIF cavity spatial filter. Measured focal distributions for the Beamlet F\#26 transport filter indicate that the pinhole loading will be as large as $120 \mathrm{GW} / \mathrm{cm}^{2}$ during the $10-\mathrm{ns}$ foot of the 21 -ns NIF pulse. Therefore, OSL experiments were designed to operate at intensities up to $500 \mathrm{GW} / \mathrm{cm}^{2}$.

Fig. 1. Fluence distribution in the drive beam.
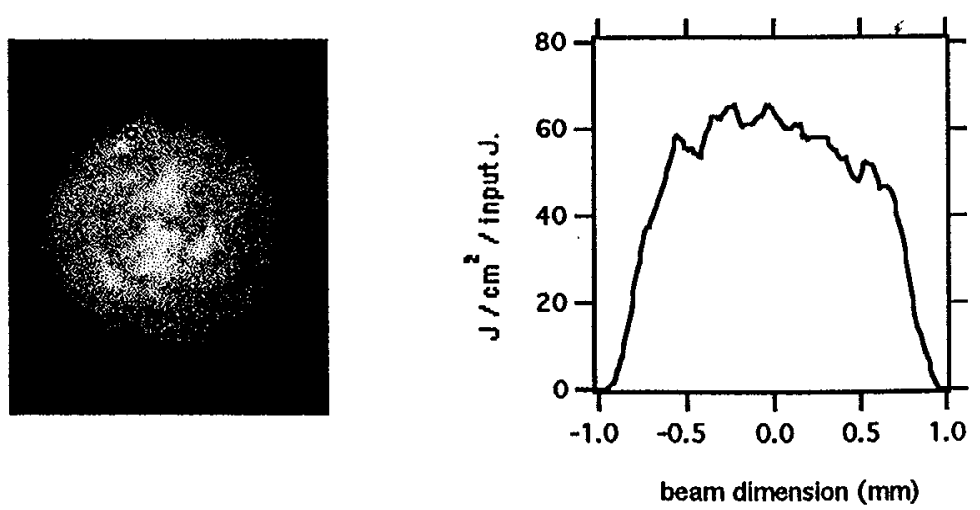
Expansion of the plasma along a horizontal axis was monitored by time-resolved two-beam interferometry. The interferometer employed a 40-ns probe beam with wavelength of either 527 or $1053 \mathrm{~nm}$ and energy, respectively, of 10 or $40 \mathrm{~mJ}$. A simplified diagram of the arrangement is shown in Fig. 2. When the $1053-\mathrm{nm}$ probe was used, the probe beam was forward propagating, but its axis was tipped in the horizontal plane by 5-7 degrees relative to the drive beam. The probe leg of the interferometer contained a pair of lenses that relayed the plane of the knife-edge sample to the mixing mirror of the interferometer. Equivalent relaying was done in the reference leg. A single lens was used to image the mixing mirror onto the slit of a streak camera. This arrangement was satisfactory when the drive beam was normally incident on a knife edge. It was also used to observe the closure of planar and 4-leaf pinholes. Interferometry with an on-axis counter-propagating 527-nm probe was used to investigate plasmas generated by drive beams that were incident at large angles on a knife edge.

Fig. 2. Time-resolved image-relayed interferometer that was used to measure the spread of plasma from knife-edge samples. Illustration shows a 1053 -nm forward-porpagating probe with incidence angle tipped relative to the drive beam. A $527-\mathrm{nm}$ counter-propagating on-axis probe was used in some experiments.

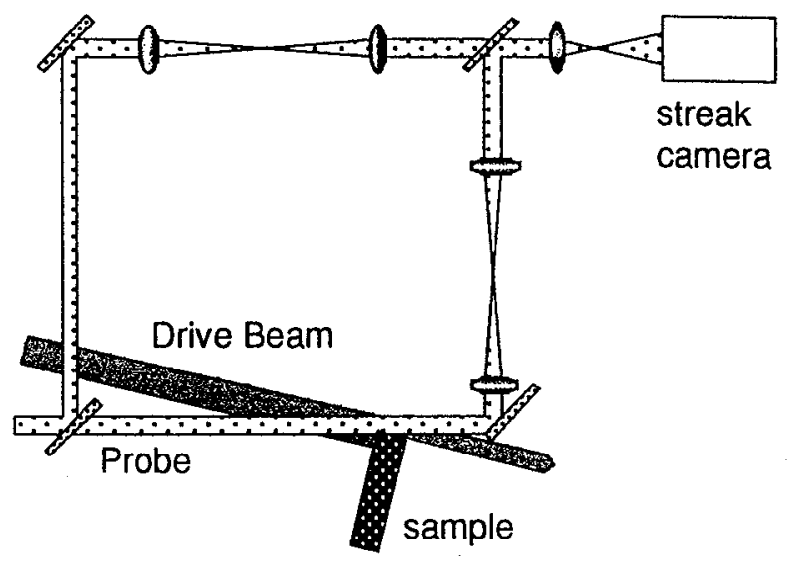

2. RESULTS

2.1 Propagation of the plasma away from the knife edge.

Each ray of the probe beam accumulates a phase governed by the electron density encountered along its path. We are interested in the velocity of propagation of particular phase shifts. Figure 3 contains interferograms that were recorded during $5-\mathrm{ns}, 130 \mathrm{GW} / \mathrm{cm}^{2}$ irradiation of knife edges that were made of carbon and tantalum. Arrival of the 5 -ns pulse is marked both by the onset of a phase disturbance and by the scattering of the drive beam into the diagnostic channel. The material dependence is visually apparent. For carbon, the phase disturbance moved rapidly across the 1.5-mm wide zone of observation. Near the carbon edge the gradient in the phase was large enough to scatter the probe beam out of the 20-mrad collection angle of the interferometer. Gradients were largely absent at the end of the observation interval. In the tantalum plasma, the phase disturbance moved more slowly. At this irradiation intensity, the phase disturbance was negligible at distances greater than $0.5 \mathrm{~mm}$. The region of large gradients near the tantalum edge persisted throughout the $25-\mathrm{ns}$ observation period after the end of the 5 ns pulse.

Fig. 3. Streaked interferograms recorded at drive intensity of $130 \mathrm{GW} / \mathrm{cm}^{2}$ for carbon (left) and tantalum (right). Time increases downward.
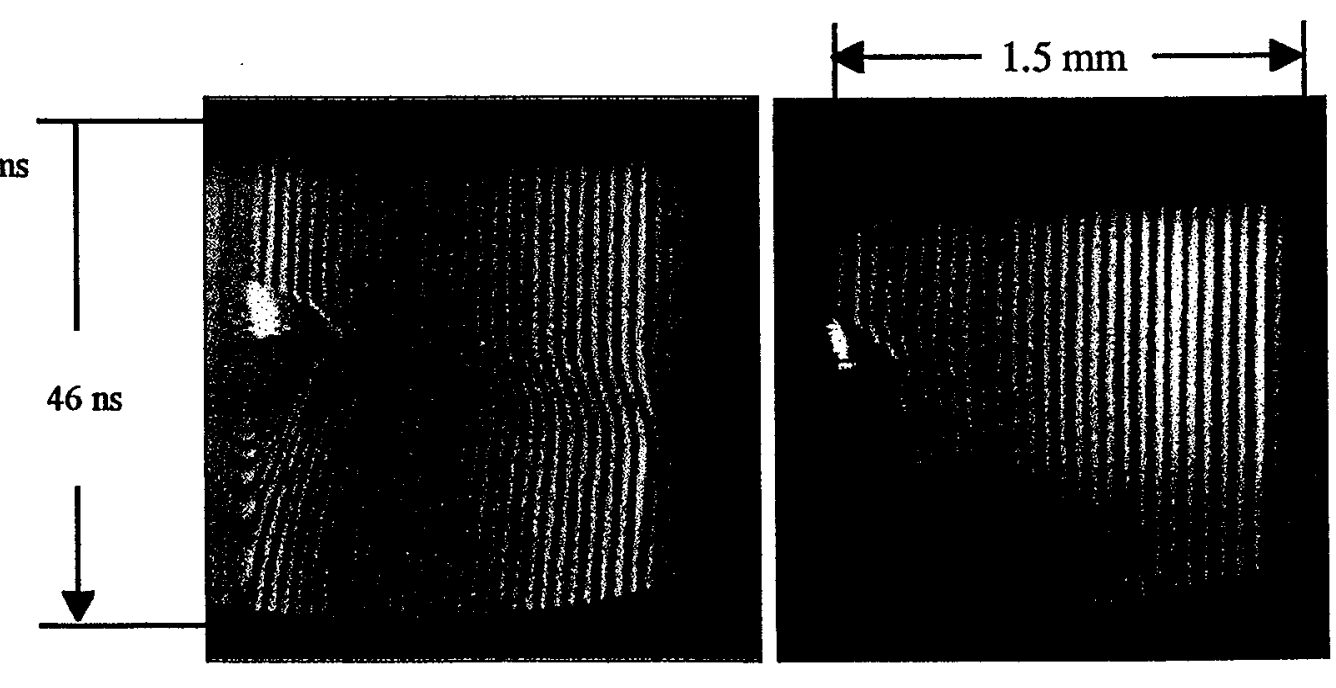
Figure 4 is a digitized version of the carbon-plasma interferogram that was shown in Fig. 3. The fringes were replaced with digitized curves, and baselines were added to show the position of the fringes prior to arrival of the drive pulse. Points were added to mark the space-time coordinates for a phase shift of $2 \pi$. For plasmas from light elements like carbon, the curve through these points was linear over a time interval considerably longer than the 5-ns irradiation. Figure 5 shows the $2 \pi$ trajectories for 5 materials. In heavy-metal plasmas, the velocity of the phase advance decreased soon after the irradiation ended. Therefore, for comparison of the different materials, we selected the velocity of the $2 \pi$ phase shift, $V_{2 \pi}$ measured during the irradiation interval.

Fig. 4. Digitized interferogram for carbon at $130 \mathrm{GW} / \mathrm{cm}^{2}$. Three fringes that could not be followed during the 5-ns irradiation are plotted as symbols. The curve indicates outward propagation of a $2 \pi$ phase shift.
Fig. 5. Trajectories of the $2 \pi$ phase shift for 5 materials that were irradiated at $130 \mathrm{GW} / \mathrm{cm}^{2}$.

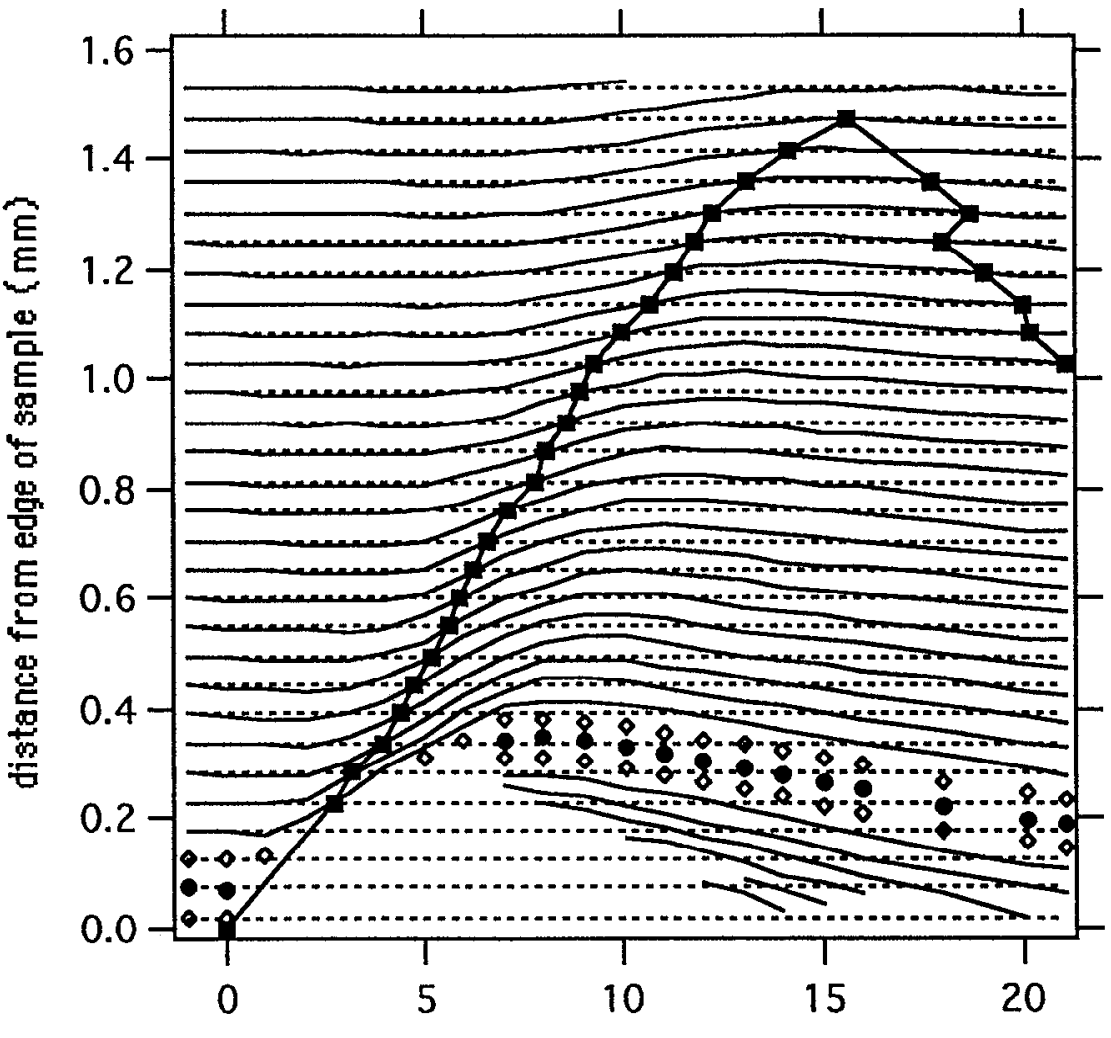

time after arrival of 5-ns pulse (ns)

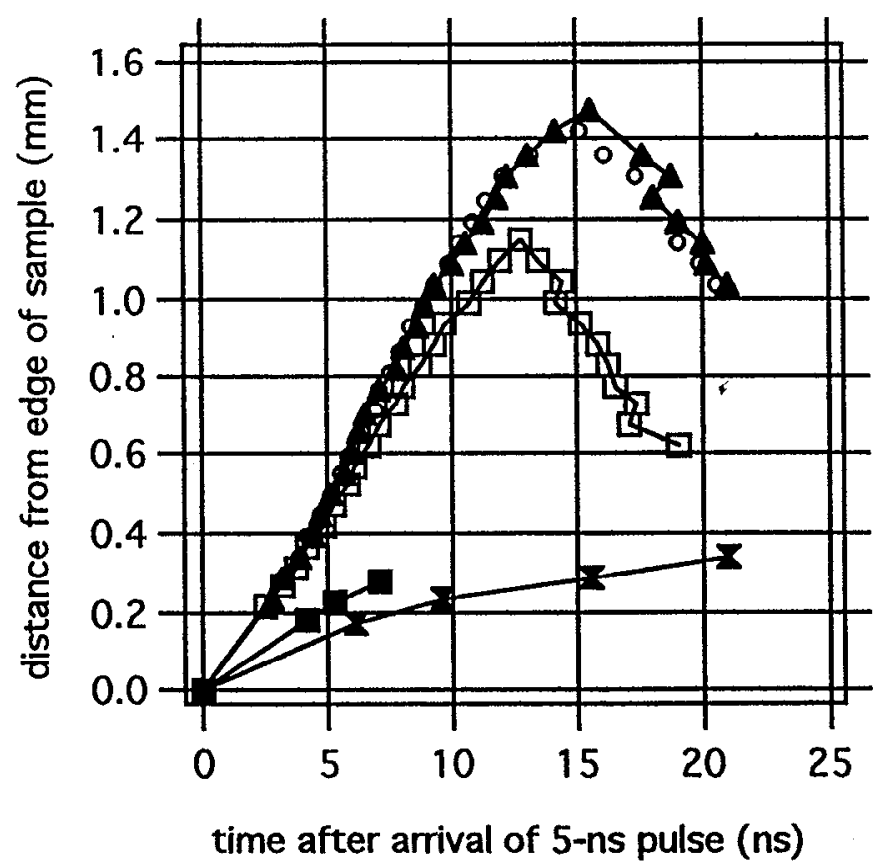


2.2. Variation of $V_{2 \pi}$ with atomic mass.

Figure 6 shows values of $V_{2 \pi}$ that were measured for four metals and carbon over a 5-ns interval of irradiaton at 130 $\mathrm{GW} / \mathrm{cm}^{2}$. The velocities in units of $\mathrm{mm} / \mathrm{ns}$ were 0.09 for carbon, 0.087 for aluminum, 0.05 for iron, 0.044 for molybdenum, and 0.028 for tantalum. Velocities for the metals were fitted to a simple power law, $\mathrm{V}=0.37 \mathrm{M}^{-0.49}$, where $\mathrm{M}$ is the atomic mass and the exponent was selected by the fitting routine. Comments on the predicted variation of $V_{2 \pi}$ with mass are provided in another paper in this session[1].

Fig. 6. Variation of $V_{2 \pi}$ with atomic mass.

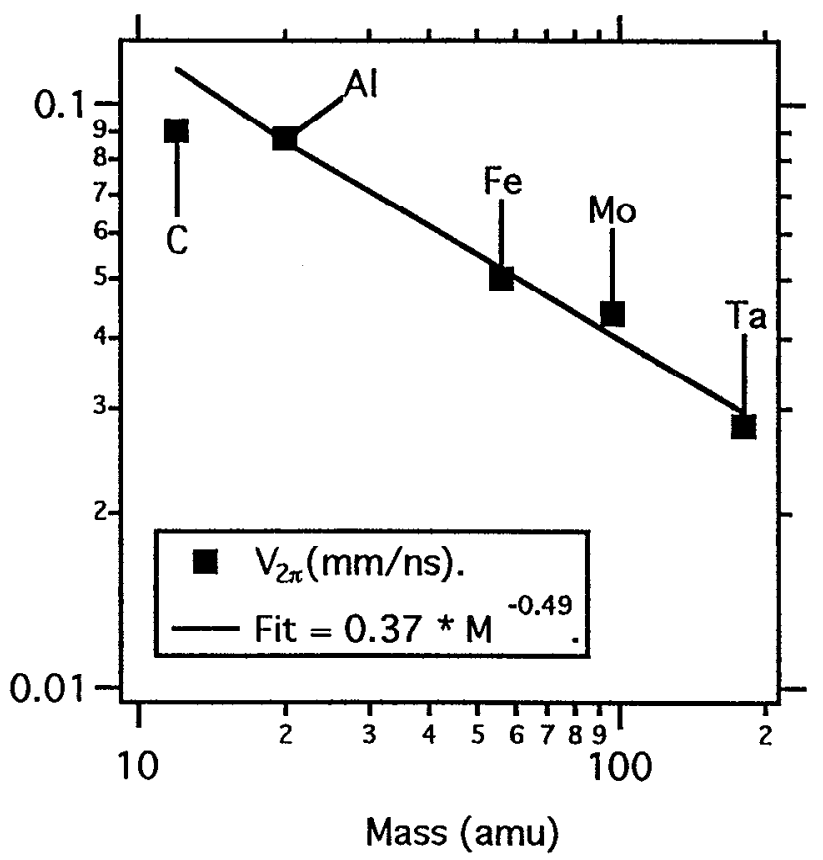

\subsection{Variation of $V_{2 \pi}$ with intensity.}

Figure 7 shows the $2 \pi$ trajectories for plasmas from a stainless steel edge that was irradiated at intensities ranging from 38 to $520 \mathrm{GW} / \mathrm{cm}^{2}$. The velocities at $5 \mathrm{~ns}$ in units of $\mathrm{mm} / \mathrm{ns}$ were 0.015 at $38 \mathrm{GW} / \mathrm{cm}^{2}, 0.046$ at $140 \mathrm{GW} / \mathrm{cm}^{2}, 0.127$ at $310 \mathrm{GW} / \mathrm{cm}^{2}$, and 0.17 at $510 \mathrm{GW} / \mathrm{cm}^{2}$. To within the precision of the data, the measured velocities increased linearly with intensity.
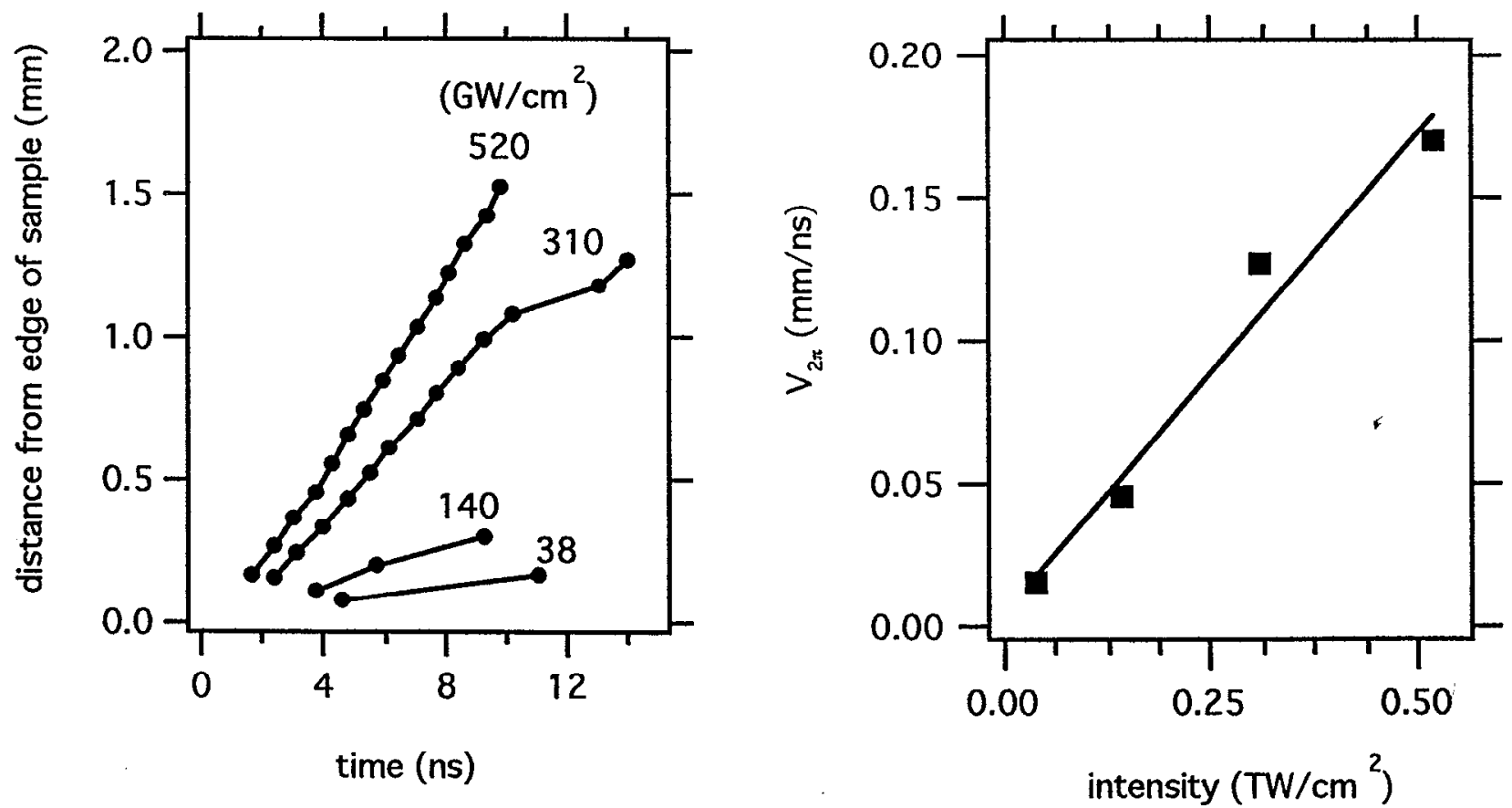

Fig. 7. Variation of $V_{2 \pi}$ for stainless steel with drive intensity. 
2.4. Variation of phase shift with distance from the edge.

The interferograms provide a measure of the variation of the phase shift along a spatial coordinate that measures distance from the edge. Figure 8 shows the phase shift measured at 1-ns intervals following the onset of the irradiation of a stainless steel edge by a 5-ns pulse at $500 \mathrm{GW} / \mathrm{cm}^{2}$. The variation was exponential to within the precision of the measurements, which is about 0.5 radians.

Fig. 8. Exponential variation of phase shift with distance from the edge of the sample.

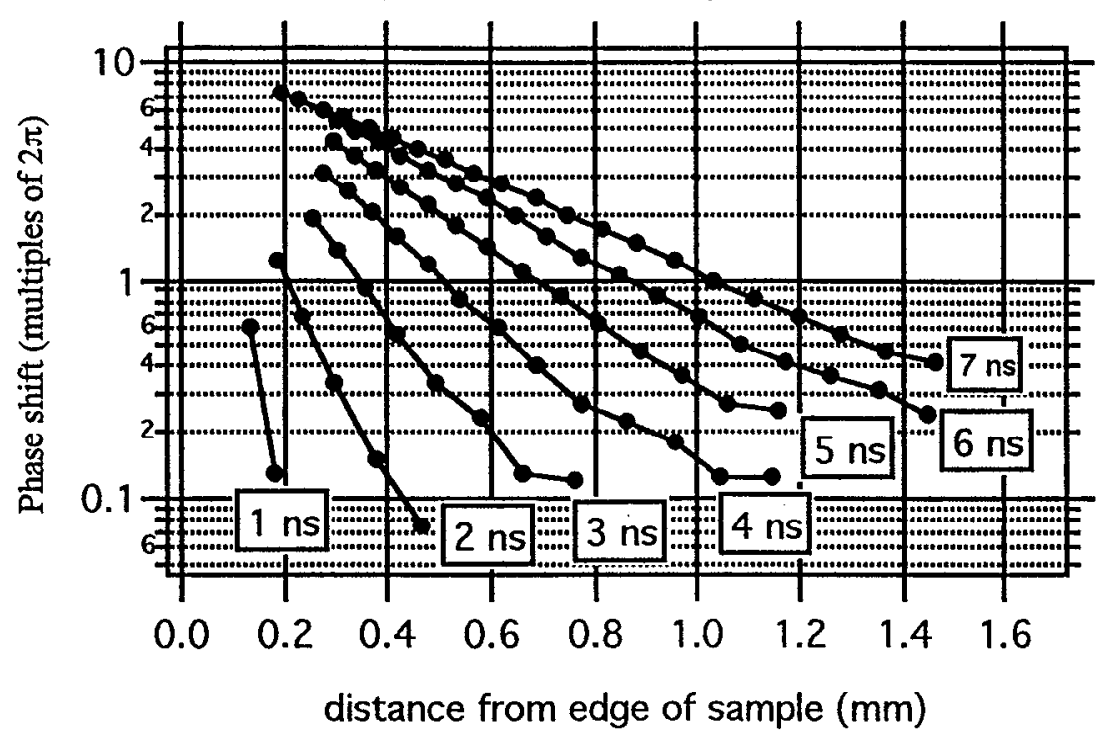

2.5. Absorbing glass.

Kurnit et al[2] have used 4-ns, 1064-nm pulses to measure the damage threshold of absorbing glass as a function of the incidence angle of the beam. At grazing incidence, the threshold of samples that were tested in air was more than $5 \mathrm{KJ} / \mathrm{cm}^{2}$. This suggests that the optimum material for conical pinholes might be either absorbing or transparent glass. A procedure for fabricating glass pinholes was presented at this conference[3].

We compared the phase shift in plasmas from Schott NG3[4] glass with that in plasmas from stainless steel and tantalum. The glass sample was an optically polished $5 \times 5 \mathrm{~cm}$ square plate with thickness of $3 \mathrm{~mm}$. It and the metal samples were irradiated by $5-\mathrm{ns}$ pulses at intensities to $270 \mathrm{GW} / \mathrm{cm}^{2}$ in a beam incident at 85 degrees relative to the normal. Plasma expansion was observed by an interferometer that used an on-axis $527-\mathrm{nm}$ probe beam. Results are shown in Fig. 9. Phase shifts in plasmas from the glass were larger than those in plasmas from steel or tantalum.

The results of the previous study [2] suggest that our test fluences were below the threshold for laser-induced damage in a grazing-incidence experiment in air. The magnitude of the permanent damage for this test in vacuum is in reasonable agreement with those results. A single test site received $5 \mathrm{P}$-polarized shots at intensities of 70, 61, 126, 190, and 240 $\mathrm{GW} / \mathrm{cm}^{2}$. These irradiations discolored the test site and made it visible to the unaided eye, but the site does not appear to have been pitted or crazed.

Fig. 9. Measured phase shifts in plasmas from absorbing glass, stainless steel and tantalum.

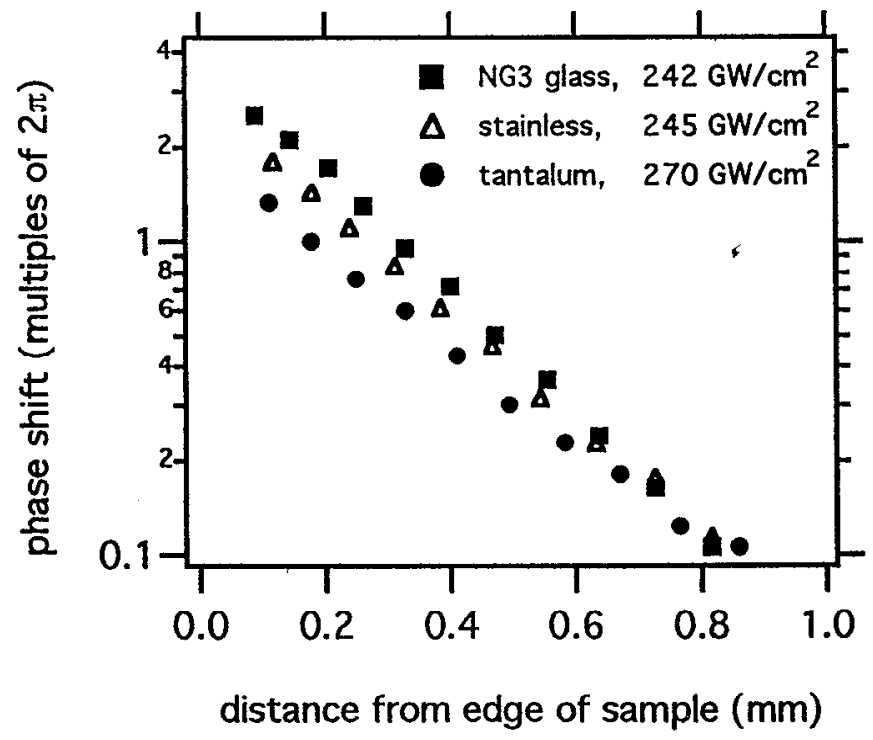


A very different result was obtained in a 5 -shot test at nearly normal incidence and at similar intensities at a different site on the NG3 sample. One half of the beam was on the edge of the sample, and the beam was incident at 5 degrees to the normal. In that experiment a semicircular notch, large enough to allow almost unobstructed passage of the beam, was punched through the edge of the 3-mm sample. The maximum test intensity of $240 \mathrm{GW} / \mathrm{cm}^{2}$ is predicted to be well above the damage threshold for incidence angle of 5 degrees[2].

\subsection{On axis convergence of plasma in a planar pinhole.}

It has been known for several years that multiple-leaf pinholes would stay open during passage of pulses that would close a planar pinhole. The simplest planar pinhole consists of a hole drilled in a thin piece of metal. It was believed that one cause of the rapid closure of planar pinholes was that plasma from every point on the perimeter converged in the center. In a multiple-leaf pinhole, the perimeter of the hole is defined by a series of plates that are distributed along the beam waist so that none of them are directly opposed to another.

We used the 1053-nm probe beam and interferometer to observe the phase shift in a circular, planar pinhole with diameter of $0.75 \mathrm{~mm}$, and in a rectangular 4-leaf pinhole with transverse dimension of about $0.9 \mathrm{~mm}$. Two of the interferograms are shown in Fig. 10. For each of these shapes, there was a measurable phase shift in the volume near the edge of the pinhole. However, the most striking feature is that the on-axis volume of the circular hole became effectively opaque while there was still transmittance in the region between the center and the edge. Such on-axis accumulation was not observed for the 4-leaf design.
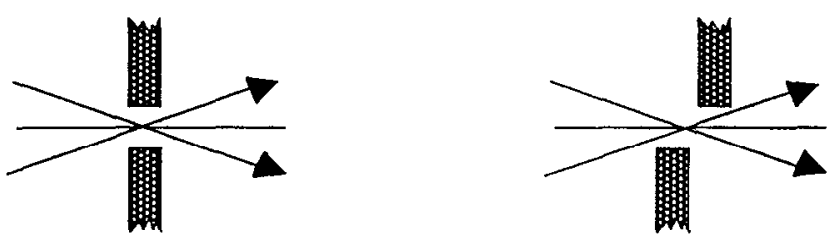

Fig. 10. The on-axis accumulation that was observed during closure of a planar pinhole (left, at $18 \mathrm{GW} / \mathrm{cm}^{2}$ ) was not observed during closure of a 4-leaf square pinhole (right, at $30 \mathrm{GW} / \mathrm{cm}^{2}$ ).
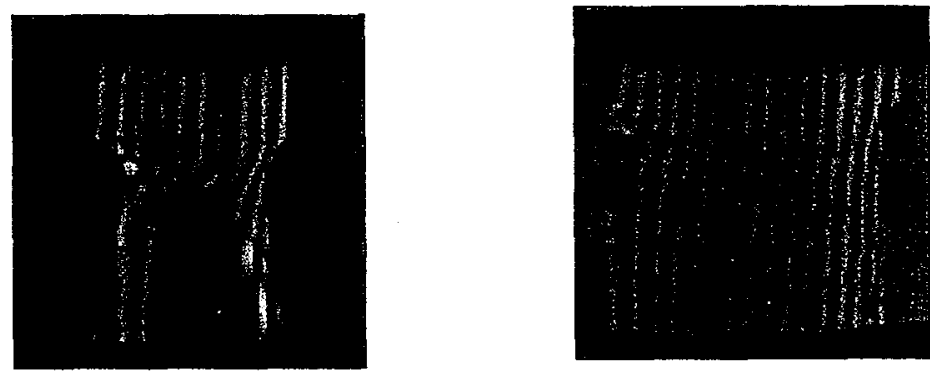

\section{ACKNOWLEDGEMENTS}

This work was performed under the auspices of the U. S. Department of Energy by the Lawrence Livermore National Laboratory, under contract W-7405-ENG-48.

\section{REFERENCES}

1. C. D. Boley, K. G. Estabrook, J. M. Auerbach, M. D. Feit, A. M. Rubenchik, "Modeling of laser knife-edge and pinhole experiments," Solid-State Lasers for Application to Inertial Confinement Fusion, June 7-12, Monterey, Califormia. 2. N. A. Kurnit and R. F. Harrison, "Measurement of the angular dependence of damage thresholds of absorbing glass for application to high-damage-threshold far-field apertures," Paper CThO35, Conference on Lasers and Electro-Optics/ International Quantum Electronics Conference, San Francisco, California, May 3-8, 1998.

3. N. A. Kurnit and S. A. Letzring, "A high-damage-threshold pinhole for glass fusion laser applications," Solid-State Lasers for Application to Inertial Confinement Fusion, June 7-12, Monterey, California.

4. NG3 is a product of Schott Glass Technologies Inc., Duryea, PA. 